\title{
Development and characterization of a gastroretentive dosage form composed of chitosan and hydroxyethyl cellulose for alendronate
}

\author{
This article was published in the following Dove Press journal: \\ Drug Design, Development and Therapy \\ 27 December 2013 \\ Number of times this article has been viewed
}

\author{
Ying-Chen Chen ${ }^{1, *}$ \\ Hsiu-O Ho ${ }^{1, *}$ \\ Chiao-Chi Chiu' \\ Ming-Thau Sheu ${ }^{1,2}$ \\ 'School of Pharmacy, College of \\ Pharmacy, Taipei Medical University, \\ ${ }^{2}$ Clinical Research Center and \\ Traditional Herbal Medicine Research \\ Center, Taipei Medical University \\ Hospital, Taipei, Taiwan \\ *These authors contributed equally \\ to this work
}

Correspondence: Ming-Thau Sheu Taipei Medical University,

$250 \mathrm{Wu}$-Hsing Street, Taipei II 0 ,

Taipei, Taiwan

Tel +886 22736 I66I ext $61 \mid 2$

Fax +886 223771942

Email mingsheu@tmu.edu.tw

\begin{abstract}
In this study, alendronate, the most commonly used biphosphonate for treating osteoporosis, was formulated as gastroretentive dosage form (GRDF) tablets to enhance its oral bioavailability. GRDF tablets were characterized with the effects of different molecular weights (MWs) of chitosan (CS) and hydroxyethyl cellulose (HEC) at various ratios on swelling, floating, and physical integrity. The CS component was formed using various acids: acetic, lactic, malic, succinic, and citric, and a high viscosity grade of HEC was selected. The results demonstrated that the swelling ratios of the formulations comprising high MW CS were lower than those of low or medium MW CS when salts were formed with any countering acids except for acetic acid. The decreasing ranking of the swelling rates was: CS-citrate $>$ CS-malate $>$ CS-lactate $>$ CSsuccinate $>$ CS-acetate. A negative correlation was found between the $\mathrm{pK}_{\mathrm{a}}$ of the respective countering acid and the swelling rate. The swelling rate was promoted if an acidic salt of CS with a lower water content was incorporated, while it became slower when tablet hardness was higher or the compression force to form tablets was increased. Although HEC did not contribute to swelling or floating, it played a role in maintaining structural integrity. A prolonged dissolution profile of alendronate GRDF tablets developed in this study was observed.

Keywords: gastroretentive dosage form, chitosan, hydrogel, hydroxyethyl cellulose, swelling, alendronate
\end{abstract}

\section{Introduction}

Because of easy administration, patient compliance, and flexible formulation, the oral dosage form still remains the most preferable route for drug delivery. The sustainedrelease dosage form offers several advantages over an immediate-release dosage form, including less frequent drug administration, prolonged periods of action resulting in maximized therapeutic efficiencies, and minimization of fluctuations in drug concentrations, with reduced incidence of adverse effects. However, the oral sustained-release dosage form is not appropriate for some drugs characterized by a narrow absorption window in the upper part of the gastrointestinal tract (GIT). This is because drugs have a short transit period (less than 6 hours in the stomach and upper small intestine) so that the sustained-release dosage form may leave the proximal GIT, and the drug is then released in non-absorbing distal segments. This would result in a short absorption phase that is often accompanied by poor bioavailability. ${ }^{1-3}$

Prolonging gastric retention is desirable for achieving the therapeutic benefit of drugs that are absorbed in the proximal part of the GIT, or are less soluble in or 
degraded by the alkaline $\mathrm{pH}$ of the lower part of the GIT. After oral administration, the gastroretentive dosage form (GRDF) could be retained in the stomach so that it may release the drug there in a sustained manner, allowing the drug to be supplied continuously to its absorption sites. ${ }^{4}$ This mode of administration will prolong the period in which the blood drug concentrations are within "therapeutic levels." Metformin $\mathrm{GR}^{\circledR}$ and ciprofloxacin GR have been marketed as a GRDF.

Currently, there are three approaches to a GRDF: 1) mucoadhesion, in which the GRDF attaches to gastric or intestinal walls in such a manner that motility is limited; 2) density modification (floating or sinking), in which the dosage form cannot leave the stomach because of its orientation to the pylorus; and 3 ) expansion (swelling), in which the GRDF expands or swells to a size that is too large to pass through the pylorus, resulting in prolonged gastric retention. ${ }^{5}$ Various combined gastroretentive mechanisms have been developed to enhance gastroretention capabilities. A sustained GRDF of ofloxacin was developed using sodium bicarbonate (SB) (floating), crospovidone, betacyclodextrin (swellable), psyllium husk, and hydroxypropyl methyl cellulose (HPMC) (bioadhesive). ${ }^{6}$ Varshosaz et $\mathrm{al}^{7}$ produced floating-bioadhesive tablets to lengthen the stay of ciprofloxacin in its absorption area, and effervescent tablets were designed using sodium carboxymethyl cellulose, HPMC, polyacrylic acid, poly(methacrylic acid), citric acid, and SB. Blends of ciprofloxacin, HPMC, swelling agents (crospovidone, sodium starch glycolate, and croscarmellose sodium), and SB were found to show more favorable swelling, floating, and drug release characteristics than those of a marketed-product (CIFRAN OD $\left.{ }^{\circledR}\right) .{ }^{8}$ We previously reported swelling and floating GRDFs of losartan, based on the combination of hydroxyethyl cellulose (HEC) and sodium carboxymethyl cellulose, ${ }^{9}$ or HEC, chitosan (CS) and SB. ${ }^{10}$ Although there are many studies attempting to develop a GRDF, the combined mechanisms of floating and swelling seem to offer greater safety for clinical uses than other approaches. ${ }^{11}$ Furthermore, the GRDF is a non-drugspecific platform and can be used with different narrow absorption window drugs.

CS is a naturally occurring, weak base polysaccharide, with a $\mathrm{pK}_{\mathrm{a}}$ value of 6.2-7.0, and is insoluble in neutral and alkaline solution. CS gels are usually formed by a chemical cross-linker of glutaraldehyde. ${ }^{12,13}$ These gels not only swell but also have intragastric-floating characteristics that prolong retention of the GRDF in the stomach. With these advantages, CS gel has been exploited widely for GRDFs. ${ }^{14-17}$
However, the chemical cross-linking agents may be toxic and have other undesirable environmental or manufacturing problems. To overcome these disadvantages, a method of using reversible physical cross-linking by ionic interaction was applied in the preparation of CS gels. In acidic media, the amine groups of the polymer are protonated, resulting in a soluble and positively charged gel, via ionic interactions with small anionic molecules, such as sulfates, citrates, and phosphates. ${ }^{18}$ Because CS is a swellable hydrogel in acidic media, and is biocompatible, biodegradable, and nontoxic, it draws considerable attention for pharmaceutical research. ${ }^{19}$ Shu and Zhu have found that there is an electrostatic interaction between sodium citrate and CS, and created citrate cross-linked CS beads and microspheres. ${ }^{20-22}$ Ionic interactions can yield hydrogels with various properties, depending on the charge density and size of the anionic agents, as well as the degree of deacetylation and the concentration of the CS. For example, CS gels possessing different water sorption profiles and crystalline structures were formed when prepared with various solubilizers (acetic, citric, formic, glycolic, lactic, malic, and propionic). ${ }^{23}$ Among aspartic, glutamic, hydrochloric, lactic, and citric acids, glutamic and aspartic CS salts provided the best colon-specific drug release. ${ }^{24}$ In this study, CS hydrogels were prepared by dissolving CS in different organic acids (acetic, lactic, malic, succinic, and citric). The hardness, swelling ratio and flotation characteristics were carefully examined to investigate the influence of countering acids on CS hydrogel.

Osteoporosis is a common disease, a result of the aging process. Currently, the major drug used to treat this disease is based on bisphosphonates. However, the limitations of bisphosphonates are local stimulation on upper digestive mucous and poor bioavailability. Davis ${ }^{25}$ mentioned that bisphosphonates would benefit from an increased residence period at the absorption site, which is the small intestine or stomach. In response to Davis' comment, this study is an attempt to develop a novel GRDF, based on the combination of CS with solubilizers (acetic, lactic, malic, succinic, and citric acid) and HEC, to prolong the gastric retention time of alendronate in the upper part of the GIT with a sustained drug release pattern. Favorably, the positive and negative charge interaction between alendronate sodium and CS would decrease the irritation of upper digestive mucous.

\section{Materials and methods Materials}

HEC 250HHX (viscosity of 3,400-5,000 cP, estimated molecular weight of $1,600 \mathrm{kDa}$ ) was supplied by Hercules 
(Wilmington, VA, USA). CS, with a molecular weight of 50-190 kDa (LCS), 190-310 kDa (MCS), and 310-375 kDa (HCS), was from Sigma-Aldrich (St Louis, MO, USA). Alendronate sodium was provided by Alcon (Bangalore, India). Acetic and malic acid were purchased from SigmaAldrich and Merck (Darmstadt, Germany), respectively. Succinic, citric, and lactic acid were from Riedel-de Haën (Seelze, Germany). All other chemicals used were reagent or pharmaceutical grade.

\section{Fabrication and characterization of GRDF tablets}

$\mathrm{CS}$, in a concentration of $1 \%$, was dissolved in various acid solutions: acetic acid (monoprotic acid, 1\%), lactic acid (monoprotic acid, 1\%), succinic acid (diprotic acid, 2\%), malic acid (diprotic acid, 2\%), and citric acid (triprotic acid, 3\%) to form CS hydrogel. Then different amounts of HEC (the ratio of CS to $\mathrm{HEC}=1 / 0,1 / 0.1$, $1 / 0.3,1 / 0.5,1 / 1 ; \mathrm{w} / \mathrm{w})$ were added to the hydrogel. Excess acid was removed by dialysis (cutoff: 6,000-8,000 Da) to neutral, and then freeze-dried. The lyophilized product was shattered and passed through a 60 -mesh sieve. A $400 \mathrm{mg}$ mixture was compressed on a Carver Laboratory Press tableting machine, without the addition of lubricant, using a flat-faced punch and die (diameter $12 \mathrm{~mm}$ ), for 6 seconds at different compression pressures $(0.25$, 0.5 , and 1 ton). Three tablets for each formulation were randomly selected and used for measuring the hardness of the tablets (PTB-311; Pharma Test, Hainburg, Germany). Additionally, part of the lyophilized powder was used to measure the water content. The nomenclature of each formulation stands for acid-CS-HEC.For example, A-LCS(1)-HEC(0) means the hydrogel is made of acetic acid (A) and LCS, and the ratio of LCS and HEC is 1:0; L-MCS(1)-HEC(0.1) means the hydrogel consists of lactic acid (L) and MCS, and the ratio of MCS and HEC is 1:0.1; M-HCS(1)-HEC(0.3) means the hydrogel includes malic acid (M) and HCS, and the ratio of HCS and HEC is 1:0.3; S-LCS(1)-HEC(0.5) means the hydrogel consists of succinic acid (S) and LCS, and the ratio of LCS and HEC is 1:0.5; C-HCS(1)-HEC(1) means the hydrogel consists of citric acid (C) and HCS, and the ratio of HCS and HEC is $1: 1$.

Physical characterizations of the tablets included: swelling ratio (based on the change of diameter), floating lag time, and floating duration, used to establish a comprehensive understanding of the floating and swelling properties of various formulations. Then, $70 \mathrm{mg}$ of the model drug, alendronate, incorporated into the optimal formulations for in vitro characterization, was prepared using the direct compression method. The mixture weight of $400 \mathrm{mg}$ was compressed on a Carver Laboratory Press tableting machine (Carver, Inc, Wabash, IN, USA), using flat-faced punches (diameter $12 \mathrm{~mm}$ ) for 6 seconds.

\section{Determination of swelling ratio}

The swelling studies were conducted using a Vankel dissolution apparatus (VK7020; Agilent Technologies Inc, Santa Clara, CA, USA). The tablets were immersed in $900 \mathrm{~mL}$ of simulated gastric fluid (SGF) without pepsin ( $\mathrm{pH}$ 1.2) at $37.0^{\circ} \mathrm{C} \pm 0.5^{\circ} \mathrm{C}$ at $100 \mathrm{rpm}$. At predetermined time points $(0.5,1,1.5,2,3,4,5,6,8$, and 12 hours), the swollen tablets were removed from the solution and immediately wiped with a paper towel to remove surface droplets. The dimensions of each formulation were measured using a dial caliper. Baumgartner et $\mathrm{al}^{26}$ concluded that swelling in the axial direction was more pronounced than in the radial direction, because compression is applied on the systems in the axial direction. The swelling ratio $\left(S_{r}\right)$ was calculated according to the following equation:

$$
S_{r}=\left(S_{t}-S_{i}\right) / S_{i}
$$

where $S_{i}$ and $S_{t}$ represent the initial diameter of the dry tablet and that of the swollen tablet at time $t$, respectively. The data represent the mean \pm standard deviation from at least three samples per formulation.

\section{Evaluation of floating capacities}

The floating capacities were examined following the procedure reported by Baumgartner et al. ${ }^{27}$ The time that the tablets required to reach the water surface (floating lag time) and the period of time during which the tablets constantly floated on the water surface (designated as floating duration) were evaluated in a dissolution vessel (Vankel, VK7020; Agilent Technologies Inc) filled with $900 \mathrm{~mL}$ of $\mathrm{SGF}$ at a temperature of $37^{\circ} \mathrm{C} \pm 0.5^{\circ} \mathrm{C}$ at $100 \mathrm{rpm}$. Triple measurements were performed for each examined formulation $(n=3)$.

\section{In vitro drug release studies}

Drug release from swollen tablets was performed in $900 \mathrm{~mL}$ of SGF at $37^{\circ} \mathrm{C} \pm 0.5^{\circ} \mathrm{C}$ at $50 \mathrm{rpm}$ for 24 hours, based on the apparatus II method (United States Pharmacopeia 29) (VK7020; Agilent Technologies Inc). The medium (5 mL) was removed at predetermined time points $(0,0.5,1,2,3,5$, 
$8,12,16$, and 24 hours) and replaced with a fresh medium of the same volume. Kuljanin et $\mathrm{al}^{28}$ developed a method to determine the amount of alendronate by the formation of the complex between non-chromophoric compound, alendronate, and iron (III) chloride in perchloric acid solution. The concentration of the complex was measured by an ultraviolet/ visible spectrophotometer (V-550; Jasco Analytical Instruments, Easton, MD, USA) at a wavelength of $300 \mathrm{~nm}$, using a $1.0 \mathrm{~cm}$ quartz cell. This method has been validated with acceptable precision and accuracy for intraday and interday assays (data not shown). The average percentage of the drug dissolved at each sampling time was calculated after correcting for the cumulative amount removed in previous samples. Each in vitro dissolution test was performed three times per formulation.


Figure I Swelling ratio of different HEC ratios to (A) LCS-acetate; (B) LCS-lactate; (C) LCS-malate; (D) LCS-succinate; and (E) LCS-citrate in simulated gastric fluid. Abbreviations: A, acetic acid; L, lactic acid; M, malic acid; S, succinic acid; C, citric acid; LCS, chitosan 50-190 kDa; HEC, hydroxyethyl cellulose. 

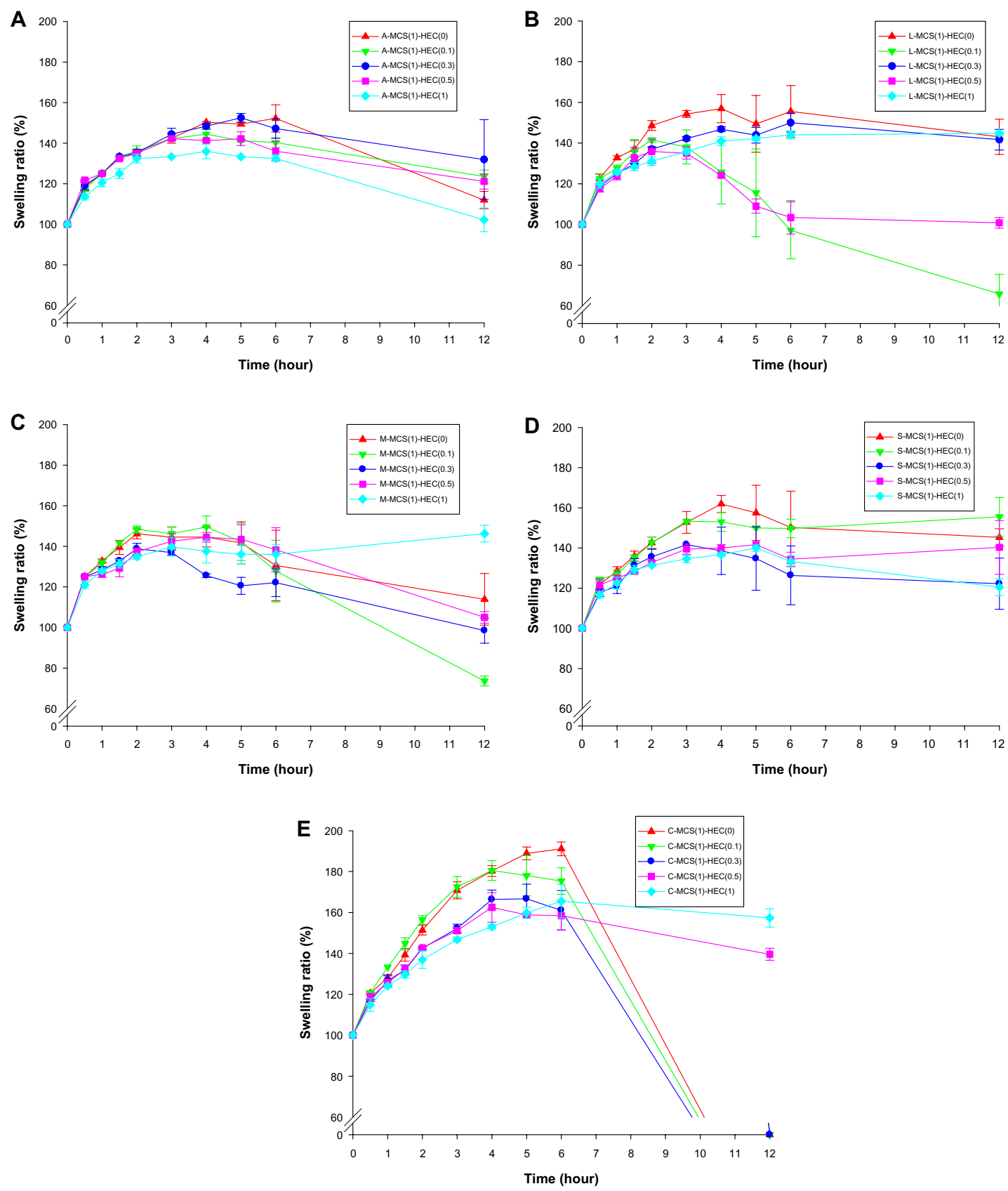

Figure 2 Swelling ratio of different HEC ratios to (A) MCS-acetate; (B) MCS-lactate; (C) MCS-malate; (D) MCS-succinate; and (E) MCS-citrate in simulated gastric fluid. Abbreviations: A, acetic acid; L, lactic acid; M, malic acid; S, succinic acid; C, citric acid; MCS, chitosan 190-310 kDa; HEC, hydroxyethyl cellulose.

swelling ratio, the swelling rate, and the integrity of tablets were influenced by the MW of CS, acidic salts of CS, and the ratio of HEC to CS. The effects of various acidic salts of different MWs of CS on the swelling ratio of GRDF tablets were compared with their respective maximal swelling ratios and the results are shown in Figure 4. It demonstrates that the maximal swelling ratios of those tablets comprised of LCS or MCS with all countering acids were higher than those of HCS except acetic acid. There were statistically significant effects of MW of CS on the swelling ratio for those GRDFs with the addition of acetic, citric, succinic, and lactic acid; however, the trend was not obvious with malic acid $(P=0.308)$. Honary et $\mathrm{al}^{29}$ also found that the swelling index rose as CS MW decreased when forming citrate cross-linked CS films. It is expected that the viscosity of CS hydrogel rises with increasing MW. Therefore, the HCS hydrogel formed initially could be too viscous to allow inward permeation of water, resulting in poor swelling ability. Zhao et a ${ }^{30}$ reported that CS dissolved in monovalent acid solutions (formic, acetic, propionic, 



Figure 3 Swelling ratio of different HEC ratios to (A) HCS-acetate; (B) HCS-lactate; (C) HCS-malate; (D) HCS-succinate; and (E) HCS-citrate in simulated gastric fluid. Abbreviations: A, acetic acid; L, lactic acid; M, malic acid; S, succinic acid; C, citric acid; HCS, chitosan 310-375 kDa; HEC, hydroxyethyl cellulose.

butyric, isobutyric, lactic, nitric, hydrochloric, and chloroacetic acid) could transform into hydrogels at $37^{\circ} \mathrm{C}$ when neutralized by a glycerophosphate solution. When dissolved in multivalent acids (sulfuric, oxalic, succinic, malic, ascorbic, phosphoric, and citric) it failed to form a gel. It was found that the type of acid used as a countering acid of CS has a substantial influence on the properties of the CS hydrogel formed; therefore, the MCS formulations were selected to examine the effect of various acidic salts and the addition of HEC at different ratios on the swelling characteristics.

The swelling rate is the change of the size of a tablet within a time period, and the change of size equivalent to a specification of its swelling ratio, in which swelling ratio and time are related to each other. The change of swelling ratio within the first 3 hours 


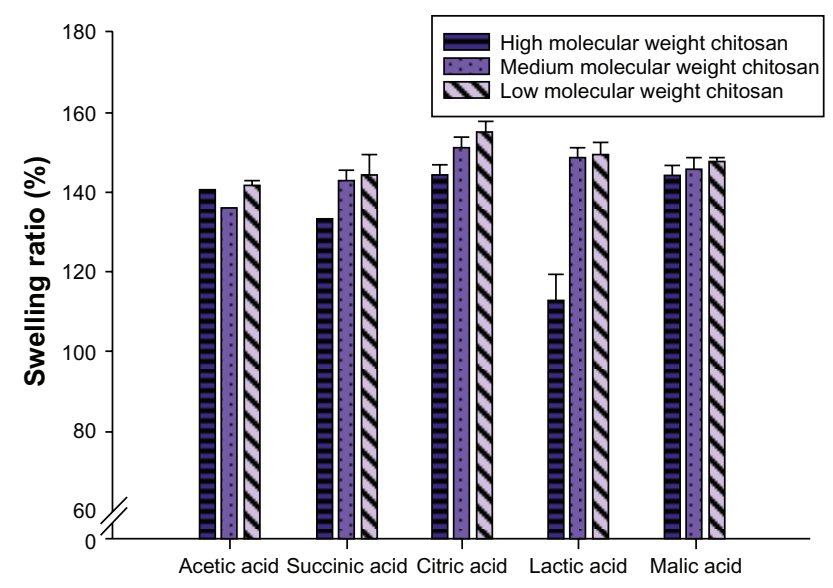

Figure 4 Swelling ratio of chitosan with various molecular weights dissolved in various acids.

was defined as the swelling rate (ie, the slope) with the unit of \%/hour.

The swelling rate (Table 1) was calculated from the swelling time profiles of Figure 2 for various acidic salts of MCS combined with different ratios of HEC. For example, the swelling rates for tablet formulations of A-MCS(1)-HEC(0), A-MCS(1)-HEC(0.1), A-MCS(1)-HEC(0.3), A-MCS(1)$\operatorname{HEC}(0.5)$, and A-MCS(1)-HEC(1) were calculated as the swelling rate of MCS-acetate. It was observed that the rank order of the decreasing swelling rates was as the following: MCS-citrate $>$ MCS-malate $>$ MCS-lactate $>$ MCSsuccinate $>$ MCS-acetate. The trend seems to be explainable by the acid dissociation constant $\left(\mathrm{pK}_{\mathrm{a}}\right)$ and water content in these formulations. Based on the equation, $\mathrm{H}^{+}+\mathrm{A}^{-} \leftrightarrow \mathrm{HA}$, the CS-salt dissociated in contact with the SGF. A smaller value of $\mathrm{pK}_{\mathrm{a}}$ causes a higher level of dissociation, more hydration, and a faster swelling rate. Further, those formulations with less water content were supposed to absorb water

Table I The average slope and water content of MCS-acetate, MCS-lactate, MCS-malate, MCS-succinate, and MCS-citrate, and their corresponding acid dissociation constant $\left(\mathrm{pK}_{\mathrm{a}}\right)$

\begin{tabular}{|c|c|c|c|c|}
\hline Formulation & Slope & $\begin{array}{l}\text { Water } \\
\text { content }\end{array}$ & Acid & $\mathbf{p K}_{\mathrm{a}}$ \\
\hline MCS-citrate & 19.56 & 7.52 & Citric acid & $\begin{array}{l}\mathrm{pK}_{\mathrm{al}}=3.15 \\
\mathrm{pK}_{\mathrm{a} 2}=4.77 \\
\mathrm{pK}_{\mathrm{a} 3}=6.40\end{array}$ \\
\hline MCS-malate & 14.00 & 13.24 & Malic acid & $\begin{array}{l}\mathrm{pK}_{\mathrm{a}}=3.4 \\
\mathrm{pK}_{\mathrm{a} 2}=5.13\end{array}$ \\
\hline MCS-lactate & 13.69 & ND & Lactic acid & $\mathrm{pK}_{\mathrm{a}}=3.86$ \\
\hline MCS-succinate & 13.79 & ND & Succinic acid & $\begin{array}{l}\mathrm{pK}_{\mathrm{al}}=4.2 \\
\mathrm{pK}_{\mathrm{a} 2}=5.6\end{array}$ \\
\hline MCS-acetate & 13.64 & $21.26 \%$ & Acetic acid & $\mathrm{pK}_{\mathrm{a}}=4.76$ \\
\hline
\end{tabular}

Abbreviations: MCS, chitosan 190-310 kDa; ND, data not done. more easily, and displayed a faster swelling rate. Ritthidej et $\mathrm{a}^{23}$ found the water sorption of CS-citrate was higher than CS-malate, CS-lactate, and CS-acetate, and the trend of water sorption can be explained by water content as shown in our study. Table 1 also shows that MCS-citrate with the lowest content of water (ie, the average content of water in C-MCS(1)-HEC(0), C-MCS(1)-HEC(0.1), C-MCS(1)$\operatorname{HEC}(0.3), \mathrm{C}-\mathrm{MCS}(1)-\mathrm{HEC}(0.5)$, and C-MCS(1)-HEC(1)) demonstrates the highest swelling rate. According to the report by Lee et al, ${ }^{31}$ the exchange of acetate of CS acetate salt with a chloride anion was expected to cause the deswelling of the brush layer due to the smaller size of the chloride anion leading to less water absorption. However, the exchange of citrate of CS citrate salt with chloride was expected to cause swelling of the brush layer as a result of the ionic cross-linking interaction between citrate and CS leading to more water absorption. Since SGF was used as the medium in our swelling study, the swelling of CS acidic salt in SGF could be recognized as the exchange of an acidic anion (acetate or citrate) with a chloride anion resulting in a slower swelling rate and less water absorption for CS acetate salt than for CS citrate salt. Our results are consistent with those reported by Lee et al. ${ }^{31}$

Originally, we attempted, using hardness data (Figure 5), to explain the integrity of the tablet after 12 hours. But the hardness values were very high for all formulations with no significant statistical difference among them. Hardness was higher for those tablet formulations containing MCS than for those containing LCS. However, among tablets containing various countering acids of MCS, the hardness of MCS-citrate was the lowest, which may result from the lower water content. To summarize, there was a negative correlation between the $\mathrm{pK}_{\mathrm{a}}$ of acids and the swelling rate (ie, slope). Water content also affected the swelling rate. However, the water content varied with the hardness. Taking MCS as an example, the correlation between $\mathrm{pK}_{\mathrm{a}}$, water content, the swelling rate, and hardness is shown in Figure 6. A smaller value of $\mathrm{pK}_{\mathrm{a}}$ led to a higher level of dissociation, resulting in more hydration and a faster swelling rate. The formulation with less water content was able to absorb water immediately, which resulted in a faster swelling rate.

\section{Effects of HEC on swelling, floating, and physical integrity}

HEC is a nonionic, water-soluble polymer that has been commonly used in pharmaceutical formulations. ${ }^{9}$ Figures 1-3 show that with the higher amount of HEC, 

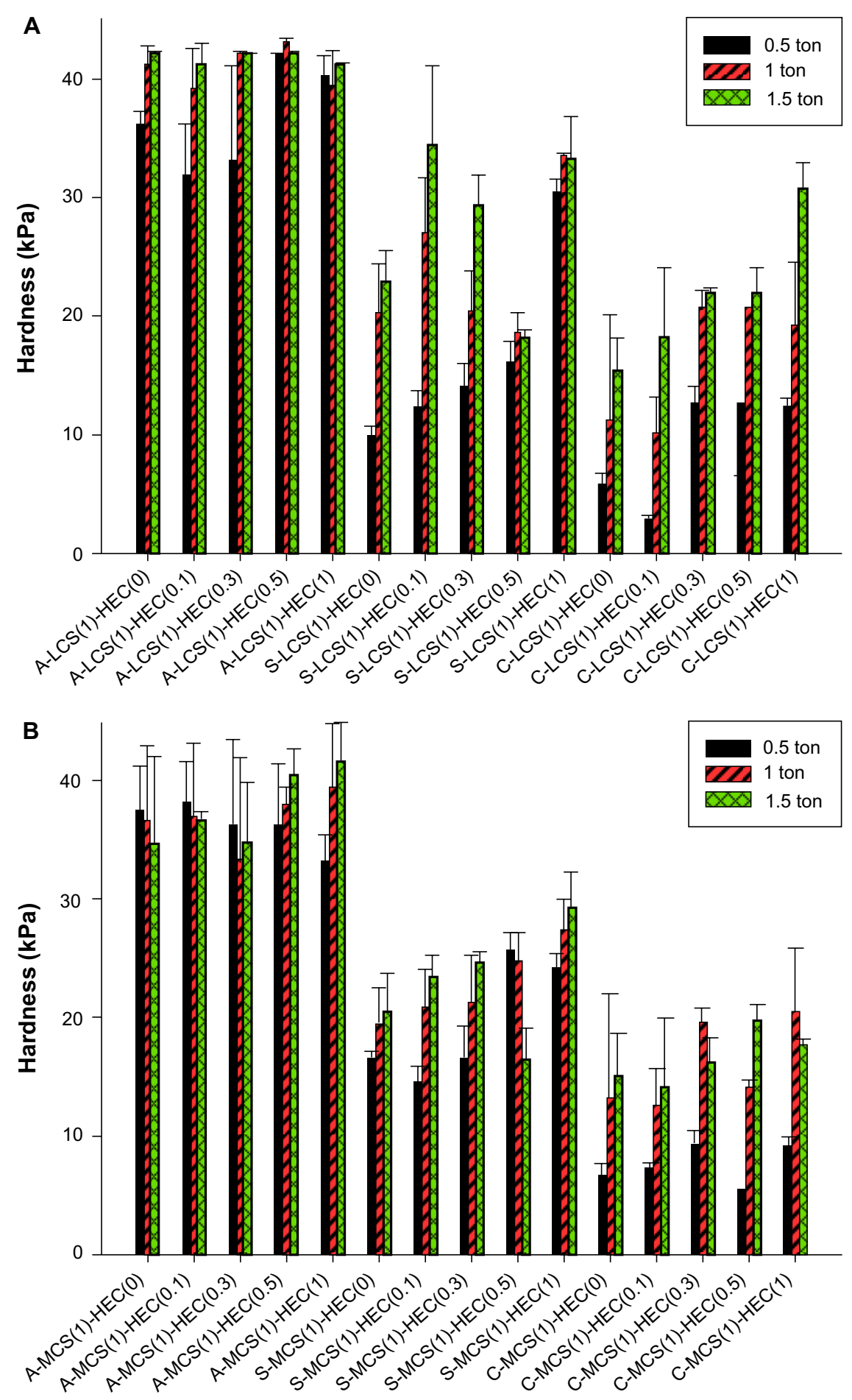

Figure 5 Hardness of tablets formulated with (A) LCS and (B) MCS.

Abbreviations: A, acetic acid; S, succinic acid; C, citric acid; LCS, chitosan 50-190 kDa; MCS, chitosan 190-310 kDa; HEC, hydroxyethyl cellulose.

the swelling ratio decreased, regardless of the type of CS. HEC is a swelling agent, but the grade of HEC 250HHX used in the study might be too viscous to retard water permeation and hence displayed poor swelling ability. In spite of using LCS, all formulations, even with the addition of HEC, were unable to float. Theoretically, HEC absorbs water and swells, resulting in a decrease of the density of the tablet and then flotation. The reason for the failure may be that we used the high viscosity form of HEC, which is relatively impermeable to water. The addition of HEC maintained structure integrity. Data from Figure 1E shows that the formulations consisting of LCS ruptured easily after 6 hours, no matter how much HEC was added. As the amount of HEC increased to more than half of CS 


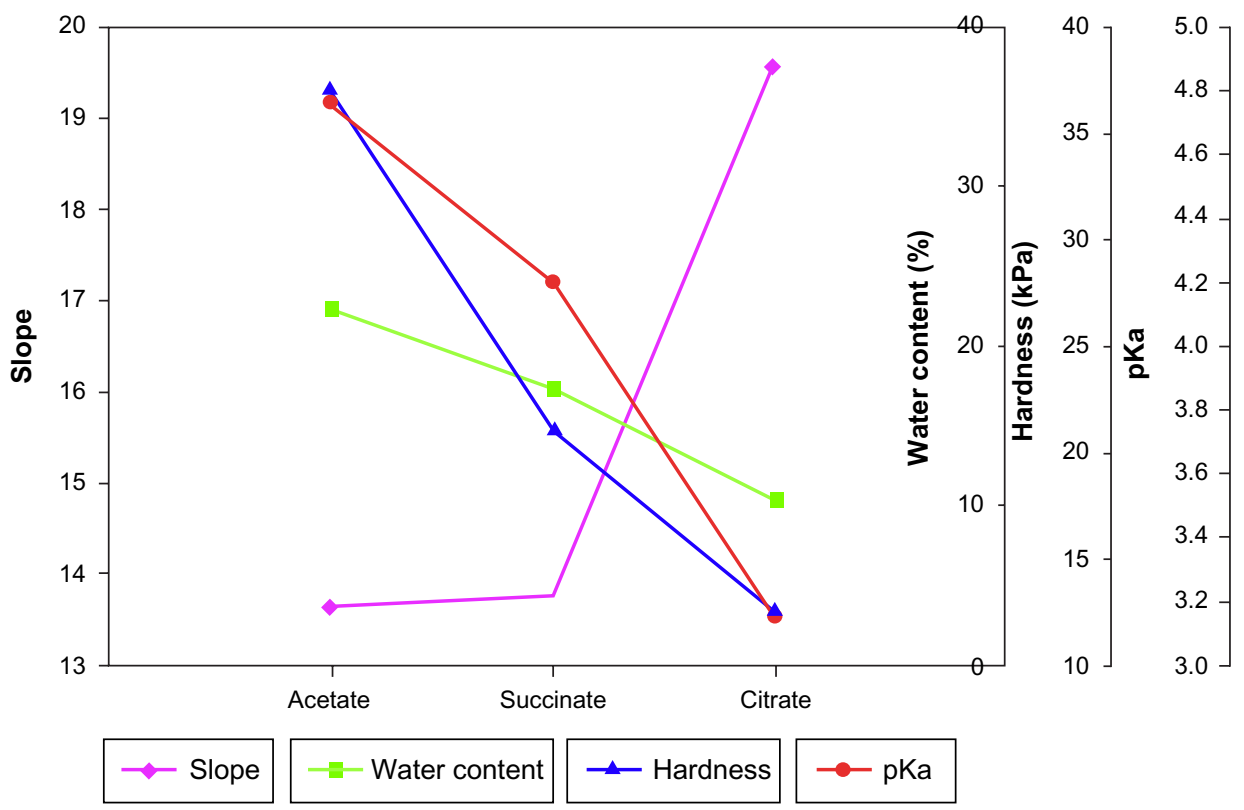

Figure 6 The correlation between $\operatorname{MCS}(\mathrm{I})-\mathrm{HEC}(0 . \mathrm{I})$-acetate/MCS(I)-HEC(0.I)-succinate/MCS(I)-HEC(0.I)-citrate and slope $(\bullet)$, water content $(\boldsymbol{\bullet})$, hardness $(\mathbf{\Delta})$, and $\mathrm{pK}_{\mathrm{a}}(\bullet)$.

Abbreviations: MCS, chitosan 190-310 kDa; HEC, hydroxyethyl cellulose.

(Figures 2E and 3E) (C-MCS(1)-HEC(0.5), C-MCS(1)$\operatorname{HEC}(1), \mathrm{C}-\mathrm{HCS}(1)-\mathrm{HEC}(0.5)$, and C-HCS(1)-HEC(1)), the tablets retained integrity for the desired period of time (12 hours). Maintaining the physical integrity of the tablet is critical in case of sustained release formulations. If it does not retain its structure, the tablet could be broken down into smaller fragments and escape from the upper part of the GIT.

\section{The effect of compression force on swelling and floating}

Although all of the formulations compressed with a pressure of 1 ton were unable to float, regardless of the type of acid or HEC added, compression force has previously been found to play an essential role in the floating and swelling characteristics, as well as in vitro drug release. ${ }^{9,10}$ Furthermore, MCS and LCS formed with acetic acid (monoprotic
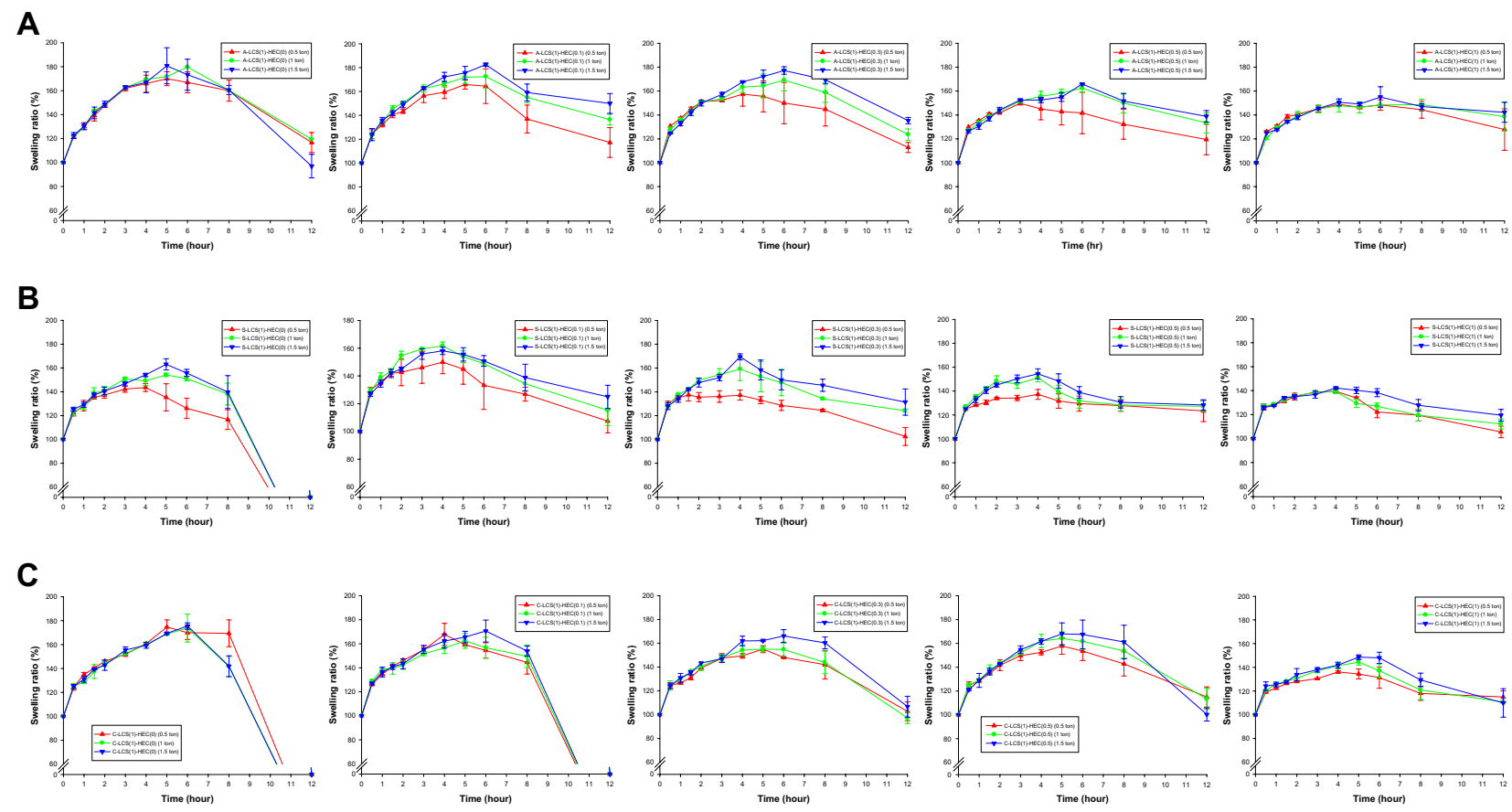

Figure 7 Swelling ratio of (A) LCS-acetate, (B) LCS-succinate, and (C) LCS-citrate, with various ratios of HEC compressed at 0.5, I, and I.5 tons. Abbreviations: A, acetic acid; S, succinic acid; C, citric acid; LCS, chitosan 50-190 kDa; HEC, hydroxyethyl cellulose. 

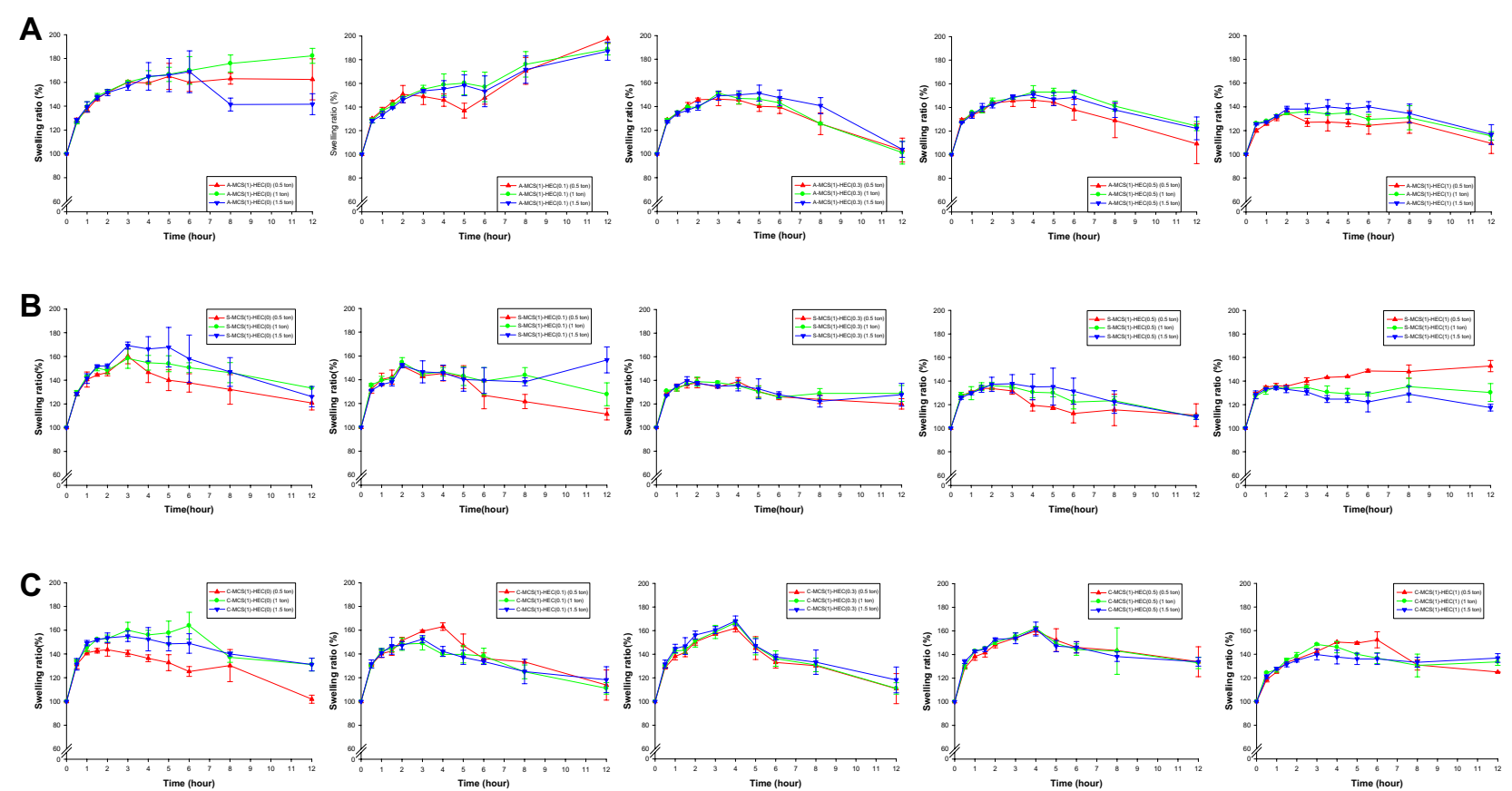

Figure 8 Swelling ratio of (A) MCS-acetate, (B) MCS-succinate, and (C) MCS-citrate, with various ratios of HEC compressed at 0.5 , I, and I.5 tons. Abbreviations: A, acetic acid; S, succinic acid; C, citric acid; MCS, chitosan 190-310 kDa; HEC, hydroxyethyl cellulose.

acid), malic acid (diprotic acid), and citric acid (triprotic acid) were chosen, with the application of different compression forces $(0.5,1$, and 1.5 tons), to seek the optimal formulation for swelling, floating, and structure integrity (Figure 7).

Figure 7 shows the swelling ratio of formulations when LCS increased with the compression force, when using the same ratio of HEC. The viscosity increased when a higher amount of HEC was used (CS/HEC $=1 / 0.5$ and 1/1), so the compression force did not show an obvious effect on the swelling ratio. The formulations S-LCS(1)-HEC(0), C-LCS(1)-HEC(0), and C-LCS(1)-HEC(0.1) were unable to maintain their structures at 12 hours, regardless of compression force, indicating the importance of HEC on physical integrity. Those formulations consisting of MCS had stronger mechanical properties independent of compaction pressure (Figure 8). However, with the addition of HEC, the swelling effect decreased because of the high viscosity and impermeability in water.
No floating occurred when using the MCS formulations, because of the higher MW, but the formulations of LCS, LCS-succinate, and LCS-citrate were able to float within 15 minutes when using a compression force of 0.5 tons. Tablets compacted at a lower pressure keep more entrapped air, decreasing the agglomerate density and allowing the tablets to float. Tablets compacted at higher pressure are usually less porous and have greater density, which prevents floating. Chen et a ${ }^{10}$ showed that tablets compressed at 0.25 tons were able to float immediately, but all denser tablets, formed at 1.0 ton compression, sank to the bottom of the dissolution vessel.

\section{In vitro drug release studies}

To achieve the goal of gastric retention, the tablets must swell to over $15 \mathrm{~mm}$ in size, to limit emptying through the pyloric sphincter (ie, swelling) ${ }^{25}$ and they must float immediately, to prevent gastric emptying and to be retained

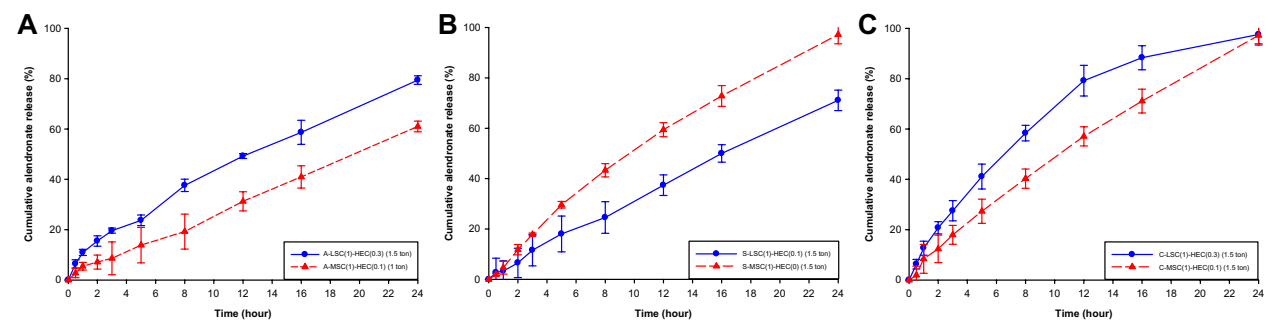

Figure 9 Dissolution profile of (A) CS-acetate, (B) CS-succinate, and (C) CS-citrate.

Abbreviations: CS, chitosan; A, acetic acid; S, succinic acid; C, citric acid; LCS, chitosan 50-190 kDa; MCS, chitosan 190-310 kDa; HEC, hydroxyethyl cellulose. 
Table 2 Release rate constant, k, and release exponent, $n$, of formulations

\begin{tabular}{lll}
\hline Formulations & $\begin{array}{l}\text { Release rate } \\
\text { constant, } \mathbf{k}\end{array}$ & $\begin{array}{l}\text { Release } \\
\text { exponent, } \mathbf{~}\end{array}$ \\
\hline A-LCS(I)-HEC(0.3) (I.5 ton) & I0.0I & 0.63 \\
A-MCS(I)-HEC(0.I) (I ton) & 4.48 & 0.77 \\
S-LCS(I)-HEC(0.I) (I.5 ton) & 4.08 & 0.89 \\
S-MCS(I)-HEC(0) (I.5 ton) & 4.92 & 1.01 \\
C-LCS(I)-HEC(0.3) (I.5 ton) & 12.07 & 0.72 \\
C-MCS(I)-HEC(0.I) (I.5 ton) & 5.80 & 0.93 \\
\hline
\end{tabular}

Abbreviations: A, acetic acid; S, succinic acid; C, citric acid; LCS, chitosan 50-190 kDa; MCS, chitosan 190-310 kDa; HEC, hydroxyethyl cellulose.

for a longer duration (ie, floating). Therefore, we selected the optimal formulation based on the following criteria: 1) tablets must expand to more than $15 \mathrm{~mm}$ within 30 minutes, and sustain the structure long enough to ensure absorption, and/or 2) the tablets should float within 15 minutes, and maintain the floatation as long as possible. The optimized formulation of A-LCS(1)-HEC(0.3) (1.5 tons), A-MCS(1)-HEC(0.1) (1 ton), S-LCS(1)-HEC(0.1) (1.5 tons), S-MCS(1)-HEC(0) (1.5 tons), C-LCS(1)$\operatorname{HEC}(0.3)$ (1.5 tons), and C-MCS(1)-HEC(0.1) (1.5 tons) were further incorporated with alendronate to evaluate in vitro drug release (Figure 9).

All formulations demonstrated sustained drug release. In the cases of the formulations A-LCS(1)-HEC(0.3) (1.5 tons) and A-MCS(1)-HEC(0.1) (1 ton), cumulative drug release at the end of 24 hours was found to be $79.45 \% \pm 1.70 \%$ and $61.04 \% \pm 2.13 \%$, respectively. C-LCS(1)-HEC $(0.3)$ (1.5 tons) and C-MCS(1)-HEC(0.1) (1.5 tons) released alendronate completely at 24 hours, and C-LCS(1)-HEC(0.3) released faster than C-MCS(1)-HEC(0.1). This might be due to the gelling properties of $\mathrm{CS}$, which could have contributed to the slower drug release. This gel increased the diffusion path length of the drug, which retarded the drug release. However, when comparing the drug release profile of S-LCS(1)$\operatorname{HEC}(0.1)$ (1.5 tons) and S-MCS(1)-HEC(0) (1.5 tons), a faster release was seen in S-LCS(1)-HEC(0.1) (1.5 tons), indicating that the reduced amount of HEC has a more profound effect than CS does on drug release in the formulations having succinic acid.

The drug release from a polymeric system occurs mostly by diffusion, as described by Fickian diffusion laws. However, in formulations containing swelling polymers, other processes, such as swelling and erosion, play an essential role in modifying the controlled drug release mechanisms. The volume expansion, caused by swelling, leads to moving diffusion boundaries, which complicates the Fickian diffusion process. ${ }^{32}$ Therefore, Korsmeyer et a ${ }^{33}$ derived a simple relationship that describes drug release from a polymeric system, as shown in the following equation:

$$
\mathrm{M}_{t}=\mathrm{kt}^{\mathrm{n}}
$$

$\mathrm{M}_{t}$ is the fraction of a drug released at time $\mathrm{t}, \mathrm{k}$ is the release rate constant, and $\mathrm{n}$ is the release exponent. The $\mathrm{n}$ value is used to characterize different releases for cylindrical shaped matrices. The value of $n$ varies, such that $n \leq 0.45$ corresponds to a Fickian diffusion mechanism, $0.45<\mathrm{n}<0.89$ to non-Fickian transport, $\mathrm{n}=0.89$ to Case II (relaxational) transport, and $\mathrm{n}>0.89$ to super case II transport.

To identify the drug release mechanism, $\mathrm{n}$ values for the different formulations were calculated using the KorsmeyerPeppas model. The values of $\mathrm{n}$ with a regression coefficient for all of the formulations are shown in Table 2. Except for the formulation S-MCS(1)-HEC(0) (1.5 tons), which did not release completely within a 24-hour period, the $\mathrm{n}$ value of other formulations ranged from 0.45 to 0.89 . These results indicate anomalous transport, which is controlled by diffusion and relaxation.

\section{Conclusion}

GRDFs benefit orally administered drugs by improving their bioavailability, therapeutic efficacy, and by possible reductions of dose. Apart from these advantages, these sustained systems offer various pharmacokinetic advantages, such as maintenance of constant therapeutic levels over a prolonged period of time, and thus a reduction of fluctuation in therapeutic levels, thus minimizing the risk of side effects. CS hydrogels were prepared by dissolving different MWs of CS in various organic acids (acetic, lactic, malic, succinic, and citric acid). There is a negative correlation between $\mathrm{pK}_{\mathrm{a}}$ and the swelling rate of CS-salt. The water content also affected the swelling rate, but varied with the hardness. Although HEC did not contribute to swelling and floating, it maintained structural integrity.

\section{Acknowledgments}

Financial support from the Center of Excellence for Clinical Trials and Research in Neuroscience (DOH 100-TD-B111-003) and the National Science Council of ROC (NSC 100-2320-B-038-004-MY3) is greatly appreciated.

\section{Disclosure}

The authors report no conflicts of interest in this work.

\section{References}

1. Streubel A, Siepmann J, Bodmeier R. Drug delivery to the upper small intestine window using gastroretentive technologies. Curr Opin Pharmacol. 2006;6(5):501-508. 
2. Nayak AK, Maji R, Das B. Gastroretentive drug delivery systems: a review. Asian Journal of Pharmaceutical and Clinical Research. 2010;3(1):2-10

3. Hoffman A. Pharmacodynamic aspects of sustained release preparations. Adv Drug Deliv Rev. 1998;33(3):185-199.

4. Chawla G, Gupta P, Koradia V, Bansal AK. A means to address regional variability in intestinal drug absorption. Pharmaceutical Technology. 2003;27(7):50-68.

5. Waterman KC. A critical review of gastric retentive controlled drug delivery. Pharm Dev Technol. 2007;12(1):1-10.

6. Chavanpatil MD, Jain P, Chaudhari S, Shear R, Vavia PR. Novel sustained release, swellable and bioadhesive gastroretentive drug delivery system for ofloxacin. Int J Pharm. 2006;316(1-2):86-92.

7. Varshosaz J, Tavakoli N, Roozbahani F. Formulation and in vitro characterization of ciprofloxacin floating and bioadhesive extendedrelease tablets. Drug Deliv. 2006;13(4):277-285.

8. Arza RAK, Gonugunta CSR, Veerareddy PR. Formulation and evaluation of swellable and floating gastroretentive ciprofloxacin hydrochloride tablets. AAPS PharmSciTech. 2009;10(1):220-226.

9. Chen RN, Ho HO, Yu CY, Sheu MT. Development of swelling/floating gastroretentive drug delivery system based on a combination of hydroxyethyl cellulose and sodium carboxymethyl cellulose for Losartan and its clinical relevance in healthy volunteers with CYP2C9 polymorphism. Eur J Pharm Sci. 2010;39(1-3):82-89.

10. Chen YC, Ho HO, Lee TY, Sheu MT. Physical characterizations and sustained release profiling of gastroretentive drug delivery systems with improved floating and swelling capabilities. Int $J$ Pharm. 2013;441(1-2):162-169.

11. Li S, Lin S, Daggy BP, Mirchandani HL, Chien YW. Effect of HPMC and Carbopol on the release and floating properties of Gastric Floating Drug Delivery System using factorial design. Int J Pharm. 2003;253(1-2):13-22.

12. Nakatsuka S, Andrady AL. Permeability of vitamin B-12 in chitosan membranes. Effect of crosslinking and blending with poly(vinyl alcohol) on permeability. J Appl Polym Sci. 1992;44(1):17-28.

13. Illum L. Chitosan and its use as a pharmaceutical excipient. Pharm Res. 1998;15(9):1326-1331.

14. Inouye K, Machida Y, Sannan T, Nagai T. Buoyant sustained release tablets based on chitosan. Drug Des Deliv. 1988;2(3):165-175.

15. Chandy T, Sharma CP. Chitosan matrix for oral sustained delivery of ampicillin. Biomaterials. 1993;14(12):939-944.

16. Patel VR, Amiji MM. Preparation and characterization of freeze-dried chitosan-poly(ethylene oxide) hydrogels for site-specific antibiotic delivery in the stomach. Pharm Res. 1996;13(4):588-593.

17. Gupta KC, Ravi Kumar MN. Drug release behavior of beads and microgranules of chitosan. Biomaterials. 2000;21(11):1115-1119.

18. Hejazi R, Amiji M. Chitosan-based gastrointestinal delivery systems. J Control Release. 2003;89(2):151-165.
19. Chang KLB, Lin J. Swelling behavior and the release of protein from chitosan-pectin composite particles. Carbohydr Polym. 2000;43(2): 163-169.

20. Shu XZ, Zhu KJ, Song W. Novel pH-sensitive citrate cross-linked chitosan film for drug controlled release. Int J Pharm. 2001;212(1):19-28.

21. Shu XZ, Zhu KJ. A novel approach to prepare tripolyphosphate/chitosan complex beads for controlled release drug delivery. Int $J$ Pharm. 2000;201(1):51-58.

22. Shu XZ, Zhu KJ. Chitosan/gelatin microspheres prepared by modified emulsification and ionotropic gelation. J Microencapsul. 2001;18(2): 237-245.

23. Ritthidej GC, Phaechamud T, Koizumi T. Moist heat treatment on physicochemical change of chitosan salt films. Int J Pharm. 2002;232(1-2): $11-22$.

24. Tengamnuay P, Sahamethapat A, Sailasuta A, Mitra AK. Chitosans as nasal absorption enhancers of peptides: comparison between free amine chitosans and soluble salts. Int J Pharm. 2000;197(1-2):53-67.

25. Davis SS. Formulation strategies for absorption windows. Drug Discov Today. 2005;10(4):249-257.

26. Baumgartner S, Lahajnar G, Sepe A, Kristl J. Quantitative evaluation of polymer concentration profile during swelling of hydrophilic matrix tablets using 1H NMR and MRI methods. Eur J Pharm Biopharm. 2005;59(2):299-306.

27. Baumgartner S, Kristl J, Vrečer F, Vodopivec P, Zorko B. Optimisation of floating matrix tablets and evaluation of their gastric residence time. Int J Pharm. 2000;195(1-2):125-135.

28. Kuljanin J, Janković I, Nedeljković J, Prstojević D, Marinković V. Spectrophotometric determination of alendronate in pharmaceutical formulations via complex formation with Fe(III) ions. J Pharm Biomed Anal. 2002;28(6):1215-1220.

29. Honary S, Hoseinzadeh B, Shalchian P. The effect of polymer molecular weight on citrate crosslinked chitosan films for site-specific delivery of a non-polar drug. Tropical Journal of Pharmaceutical Research. 2010;9(6):525-531.

30. Zhao QS, Cheng XJ, Ji QX, Kang CZ, Chen XG. Effect of organic and inorganic acids on chitosan/glycerophosphate thermosensitive hydrogel. Journal of Sol-Gel Science and Technology. 2009;50(1): 111-118.

31. Lee HS, Yee MQ, Eckmann YY, Hickok NJ, Eckmann DM, Composto RJ. Reversible swelling of chitosan and quaternary ammonium modified chitosan brush layers: effect of $\mathrm{pH}$ and counter anion size and functionality. J Mater Chem. 2012;22(37):19605-19616.

32. Siepmann J, Peppas NA. Modeling of drug release from delivery systems based on hydroxypropyl methylcellulose (HPMC). Adv Drug Deliv Rev. 2001;48(2-3):139-157.

33. Korsmeyer RW, Gurny R, Doelker E, Buri P, Peppas NA. Mechanisms of solute release from porous hydrophilic polymers. Int $J$ Pharm. 1983;15(1):25-35.
Drug Design, Development and Therapy

\section{Publish your work in this journal}

Drug Design, Development and Therapy is an international, peerreviewed open-access journal that spans the spectrum of drug design and development through to clinical applications. Clinical outcomes, patient safety, and programs for the development and effective, safe, and sustained use of medicines are a feature of the journal, which
Dovepress

has also been accepted for indexing on PubMed Central. The manuscript management system is completely online and includes a very quick and fair peer-review system, which is all easy to use. Visit http://www.dovepress.com/testimonials.php to read real quotes from published authors. 